OPEN ACCESS

Edited by:

Nader Hanna,

University of Maryland, Baltimore,

United States

Reviewed by:

Ila Pant,

Icahn School of Medicine at Mount

Sinai, United States

Gang Sun,

People's Liberation Army General

Hospital, China

*Correspondence:

Yong Yuan

yongyuan@scu.edu.cn

${ }^{\dagger}$ These authors have contributed equally to this work

Specialty section:

This article was submitted to Gastrointestinal Cancers,

a section of the journal

Frontiers in Oncology

Received: 30 May 2021 Accepted: 24 September 2021 Published: 18 October 2021

Citation:

Zhou J, Sun S, Luan S, Xiao $X$ Yang $Y$, Mao $C$, Chen L, Zeng $X$, Zhang $Y$ and Yuan $Y$ (2021) Gut Microbiota for Esophageal Cancer: Role in Carcinogenesis and Clinical Implications.

Front. Oncol. 11:717242. doi: 10.3389/fonc.2021.717242

\section{Gut Microbiota for Esophageal Cancer: Role in Carcinogenesis and Clinical Implications}

\author{
Jianfeng Zhou ${ }^{1 \dagger}$, Shangwei Sun ${ }^{1 \dagger}$, Siyuan Luan ${ }^{1}$, Xin Xiao ${ }^{1}$, Yushang Yang ${ }^{1}$, \\ Chengyi Mao ${ }^{1}$, Longqi Chen ${ }^{1}$, Xiaoxi Zeng ${ }^{2}$, Yonggang Zhang ${ }^{3,4}$ and Yong Yuan ${ }^{1 *}$ \\ ${ }^{1}$ Department of Thoracic Surgery, West China Hospital, Sichuan University, Chengdu, China, ${ }^{2}$ West China Biomedical Big \\ Data Center, West China Hospital, Sichuan University, Chengdu, China, ${ }^{3}$ Department of Periodical Press, National Clinical \\ Research Center for Geriatrics, West China Hospital, Sichuan University, Chengdu, China, ${ }^{4}$ Nursing Key Laboratory of \\ Sichuan Province, West China Hospital, Sichuan University, Chengdu, China
}

Esophageal cancer $(E C)$ is a common malignant tumor of the upper digestive tract. The microbiota in the digestive tract epithelium comprises a large number of microorganisms that adapt to the immune defense and interact with the host to form symbiotic networks, which affect many physiological processes such as metabolism, tissue development, and immune response. Reports indicate that there are microbial compositional changes in patients with EC, which provides an important opportunity to advance clinical applications based on findings on the gut microbiota. For example, microbiota detection can be used as a biomarker for screening and prognosis, and microorganism levels can be adjusted to treat cancer and decrease the adverse effects of treatment. This review aims to provide an outline of the gut microbiota in esophageal neoplasia, including the mechanisms involved in microbiota-related carcinogenesis and the prospect of utilizing the microbiota as EC biomarkers and treatment targets. These findings have important implications for translating the use of gut microbiota in clinical applications.

Keywords: microbiota, esophageal cancer, carcinogenesis, biomarker, therapeutic complication

\section{INTRODUCTION}

Esophageal cancer (EC) is one of the most common cancers and is a primary health burden worldwide (1). Globally, among all cancers, EC ranked tenth in incidence (>604,000 new cases) and sixth in mortality ( $>544,000$ deaths) in $2020(2,3)$. Moreover, the incidence of EC in Asian countries is 2.1-16.9/100,000 (4), which is higher than in other regions of the world. EC is composed of esophageal squamous cell carcinoma (ESCC) and esophageal adenocarcinoma (EAC) (1), which are more prevalent in developing and developed countries, respectively (5). As with many diseases, several genetic and environmental factors play key roles in the formation and progression of EC (6). The exact cause of EC is unknown, but smoking and heavy drinking have been proven to be

\footnotetext{
Abbreviations: BE, Barret's esophagus; EAC, esophageal adenocarcinoma; EC, esophageal cancer; ESCC, esophageal squamous cell carcinoma; GERD, gastroesophageal flux disease; LPS, lipopolysaccharide; NOD, nucleotide-binding oligomeric domain; RE, reflux esophagitis; rRNA, ribosomal RNA; TLR, Toll-like receptors; ROS, reactive oxygen species.
} 
important causes $(7,8)$. Recently, Kamangar et al. reported the impact of hot tea on esophageal carcinogenesis (9). These studies reveal that the environment plays a significant role in EC.

Gut microbiota principally refers to the microorganisms (mainly comprising bacteria) that dwell in the digestive tracts of humans (10), principally including the esophageal, oral, and intestinal microbiota (11-13). In the past few years, the role of gut microbiota in cancer environmental factors has become an increasing concern. Interestingly, a recent study reported that infectious etiology accounts for approximately $15 \%$ of all cancer cases, such as gastric cancers caused by Helicobacter pylori and hepatocellular carcinomas caused by hepatitis viruses (14). In addition to specific infectious microorganisms, several researchers have started to focus on the symbiotic microbial community, which may lead to tumorigenesis. In the case of the esophagus, these microorganisms are important to esophageal physiology such as metabolism (15) and immune maturation (16), and changes in their relative abundance can disrupt their balanced interaction with the esophagus, leading to esophageal diseases, such as EC (17). In this review, we summarize the relationships between gut microbiota and EC and evaluate their potential for clinical applications.

\section{MICROBIOTA IN ESOPHAGEAL CANCER AND DETECTION METHODS}

Human studies currently use comparative metagenomic approaches to explore the role of microbiota in EC. However, in the 1980s, traditional bacterial culture methods were still dominant in microbiology research (18), and early studies reported that there are a substantial number of microorganisms in many microenvironments that cannot be cultured (19).

Later, metagenomic studies have used non-culture methods to characterize the diversity of microbial flora, and these methods have higher sensitivity and specificity (20). 16S ribosomal RNA (16S rRNA) sequences have emerged because they can detect the previously unknown species, indicating that cultivation-based methods find less than $1 \%$ of the microbiota in samples (21). At present, the most commonly used technique in metagenome research is also the sequencing of the $16 \mathrm{~S}$ rRNA gene, which is located in the highly conserved region of all bacteria (22). After amplification with universal primers, the read sequence is compared with the database of known 16S rRNA gene sequences, which can be used to classify and identify the bacteria in the sample (23), thus, $16 \mathrm{~S}$ rRNA sequencing technology notably reduces the cost of identifying the composition of esophageal microorganisms, making large-scale research possible (24).

Recently, researchers are no longer satisfied with measuring just a tiny piece of a microbial genome. Metagenomic sequencing, which sequenced the whole genome of microorganisms, emerged. In 2002, Breitbart et al. first used environmental metagenomic sequencing to demonstrate that seawater contains over 5,000 different viruses (25). Metagenomic sequencing can provide complete information on the whole genome by randomly fragmenting all the DNA of the microbiota in samples into small segments; then, they are assembled together based on the overlapping ends into longer fragments (26). Moreover, the complete genome information can be used to metabolic capacities of the microbiota in the samples, which makes it is more valuable than the $16 \mathrm{~s}$ rRNA sequencing.

The common feature of these two methods is that they use sequencing technology to detect the DNA sequence of microorganisms in the samples, which they compare with the database of known sequences to determine the existence and abundance of specific microorganisms (22). Recently, a large number of human metagenomic sequencing or 16S rRNA sequencing studies have been conducted to describe the EC microflora of esophageal tissues and stool and saliva samples (20). A growing number of studies have revealed that the gut microbiota related to EC differs from the microbiota of healthy subjects $(27-30)$.

\section{Esophageal Microbiota in Esophageal Adenocarcinoma}

It is well known that gastroesophageal flux disease (GERD) complicating Barrett's esophagus (BE) eventually leads to EAC (5). Approximately $10 \%$ of GERD patients develop BE, and $1 \%$ of them will most likely develop EAC (31). In a meta-analysis, the occurrence of GERD symptoms at least weekly increased the risk of EAC approximately five times (32).

In this context, several studies have investigated the esophageal microbial community in patients with BE. For example, Macfarlane et al. found distinct differences in esophageal microbiota samples between BE patients and normal individuals (33). The study showed that more types of bacteria were isolated from patients with $\mathrm{BE}$ than from patients without BE; for example, Campylobacter was abundant in patients with $\mathrm{BE}$, whereas it was not identified in normal individuals (33). This indicates that patients with BE had higher microbial diversity. Furthermore, using $16 \mathrm{~S}$ rDNA technology, Yang et al. investigated the esophageal microflora of patients with a normal esophagus, reflux esophagitis (RE), and BE. According to the sequencing results, they divided the esophageal microflora into type I and type II microflora. Comprised mainly of Gram-positive bacteria such as Firmicutes, type I microflora are related to the normal esophagus. Type II microflora are mainly associated with RE and $\mathrm{BE}$ and comprise a large number of Gram-negative anaerobic/aerobic microorganisms such as Bacteroides, Proteus, Clostridium, and Spirillum (27). The transition from Gram-positive aerobic microflora to Gram-negative anaerobes may be related to changes in the microenvironment and a disease state (27). Moreover, Liu et al. also compared the $16 \mathrm{~S}$ rDNA results of esophageal bacteria in patients with $\mathrm{RE}$ and $\mathrm{BE}$ and normal individuals, and found that Clostridium welchii, Prevotella, Neisseria, and Clostridium were more common in $\mathrm{RE}$ and BE patients than in normal people (34). In addition, Yang et al. showed that the number of bacteria in the normal, esophagitis, and $\mathrm{BE}$ groups was similar and the change was the relative abundance of bacteria (35). These studies suggest that the 
composition of esophageal microflora in normal esophagus, RE, and $\mathrm{BE}$ is different, indicating that esophageal diseases may be associated with the microflora structure.

In contrast to GERD and BE, the microbial diversity of the esophagus with EAC decreased compared with normal controls because of the abundance of Lactobacillus and some other bacteria (36). The development of EAC and the lactic acid produced by Lactobacillus may acidify the internal environment of the esophagus. In addition, hydrogen peroxide, a toxic product of these bacteria, may directly inhibit the growth of other bacteria, making the lower part of the EAC esophagus to be dominated by Lactobacillus (36). Therefore, abundant Lactobacillus may be applicable to early EAC detection. In a study published in 2019, samples were collected from 16 normal individuals, 14 patients with non-dysplastic BE, 10 patients with dysplastic $\mathrm{BE}$, and 4 patients with $\mathrm{EAC}$, and the microbiota changes were apparent in cases of low-grade dysplasia and EAC, where the proportion of Firmicutes (mainly Streptococcus) in lowgrade dysplasia was higher than that in adenocarcinoma (37).

Apart from the differences in microbial diversity, studies have also focused on the functions of different microorganisms. For instance, Lopetuso et al. found distinct functions for both BE and EAC groups. On the one hand, the microorganisms have a high tendency for replication and repair in $\mathrm{BE}$, while on the other hand, there is an upregulated potential for energy, replication, and signaling metabolism and a downregulation trend of the fatty acid biosynthesis and nitrogen and D-alanine pathways in EAC (38). Overall, the EAC microbiota and associated precancerous lesions exhibit an apparent compositional and metabolic function shift compared with the microbiota of healthy individuals, reflecting a different ecological microenvironment of the esophagus in patients with EAC. Hence, it is plausible to suggest that the change in microflora may be related to the development of EAC.

\section{Esophageal Microbiota in Esophageal Squamous Cell Carcinoma}

It is now well established that esophageal dysplasia is a precursor for the majority of ESCC (39). As most cases of ESCC begin with malignant transformation of dysplasia, a growing number of studies have attempted to detect the microbiota of esophageal dysplasia and ESCC. A study published in 2014 gathered samples from 192 subjects without esophageal squamous dysplasia and 142 patients with esophageal squamous dysplasia and showed a significant difference in microbiota between these two groups, where lower microbial richness was associated with the presence of esophageal squamous dysplasia (40). Moreover, Li et al. found a significant increase in $H$. pylori infection in esophageal tumor tissues (including ESCC and samples adjacent to the ESCC) compared with that in non-tumor tissues. The $16 \mathrm{~S}$ rRNA-positive rate of H. pylori in ESCC, samples adjacent to the ESCC, and normal samples were $62.5,74.1$, and $26.7 \%$, respectively (41). This study suggested that the high level of $H$. pylori in the esophagus may be related to the development of ESCC. Gao et al. found a special infection of Porphyromonas gingivalis in the esophageal tissues of patients with ESCC, but the infection was unnoted in healthy tissues of the control group, supporting the vital pathogenic role of $P$. gingivalis in ESCC (28). In addition, Shao et al. found that the microbial environment of ESCC tissues was primarily composed of Firmicutes, Bacteroidetes, and Proteobacteria. ESCC tumor tissues contained more Fusobacterium and less Streptococcus than nontumor tissues (42). These differences offer proof of the key role of the microbiota in carcinogenesis and reveal some underlying communities of microorganisms that are likely carcinogenic.

In addition, microbial imbalances can induce further systemic metabolic changes $(43,44)$. For instance, $\mathrm{Li}$ et al. found that compared with the healthy control group, the pathways related to the metabolism of cysteine, methionine, fructose, galactose, and starch, as well as the pathways related to DNA repair and recombination, protein translation, chromosomal dynamics, and peptidase activity were upregulated in the ESCC group (45). Moreover, a study published in 2021 in which samples were collected from 18 patients with ESCC and 11 normal subjects revealed that the ESCC microbiota had altered nitrate and nitrite reductase activities compared with the normal control group (46). Animal studies in ESCC mice also showed changes in esophageal metabolism; for instance, Cheung found a decrease in Pasteurellales and upregulated metabolic pathways relevant to carbohydrate and lipid metabolism in a xenograft mouse model of ESCC cells (47). Consequently, there is a reason to believe that changes in these metabolic pathways caused by the ESCC microbiota might be closely related to the appearance and development of esophageal tumors. Therefore, we believe that the change from normal microbiota to ESCC microflora is closely related to the occurrence and development of ESCC.

\section{Oral and Intestinal Microbiota in Esophageal Cancer}

In addition to the microbiota in the esophagus itself, the oral and intestinal microbiota are vital in the development of the tumor. For instance, Snider et al. found that at the phylum level, there were distinctly enhanced relative loads of Firmicutes and decreased relative loads of Proteobacteria in BE patients (29). This reveals that the oral microbiome in $\mathrm{BE}$ patients was significantly changed, which might be related to the progress of BE. Furthermore, Peters et al. found that the composition of oral microflora can reflect the potential risk of EC; Neisseria and Streptococcus pneumoniae were positively related to the presence of EAC, and a load of $P$. gingivalis was consistent with a high risk of ESCC (48). In addition, a study published in 2015 collected saliva samples from 87 incident and histopathologically diagnosed ESCC cases, 63 dysplasia cases, and 85 non-disease cases and reported the existence of obvious oral microbiota shifts from normal oral microbiota to ESCC microflora (30). Therefore, proposing a prediction model for the change in the corresponding microflora may provide clues regarding the early detection and diagnosis of EC. In addition, Qian et al. collected saliva from 20 patients with ESCC and 21 healthy controls and found that the healthy control group was positively related to Fusobacterium and Porphyromonas, while the susceptibility of ESCC was possibly correlated to Actinomyces and Atopobium (49). Futhermore, a study published in 2019 by Kageyama 
reported that there was more $P$. gingivalis in the saliva of EC patients relative to that in the control subjects (50); $P$. gingivalis might play the same role in EC as hepatitis viruses in hepatocellular carcinomas. In addition, Zhao et al. found that in 39 EC patients, the most significantly increased taxa were Firmicutes, Negativicutes, and Selenomonadales, compared with 51 healthy subjects (11). Above all, the oral microbiota in EC patients exhibit a shift relative to the microbiota of healthy individuals, and these changes lay the foundation for the use of oral microbiota as biomarkers for the early diagnosis of EC.

In addition, several studies have shown the intestinal microbial structure in patients with EC. For instance, Tanaka et al. reported that obligate anaerobes, such as the Clostridium coccoides and Bacteroides fragilis groups, were predominant in patients with esophageal tumors (51). Moreover, a study published in 2015 collected stool samples from 31 subjects with advanced EC revealed that the Clostridium leptum subgroup and Clostridium coccoides group dominated the community of gut microbes in EC participants (12). These studies suggested that the gut microbiota related to EC was characteristic, enabling them to be latent biomarkers for early EC diagnosis.

\section{MECHANISMS IN CARCINOGENESIS}

The number of genes in the gut microflora is approximately 100 times that in the human genome (52). Although the microbiota is most dense in the lower intestine, its influence on host immunity extends beyond the lower intestine (53). These genes provide complex tools that enable microbiota to use digestive tract substances to adapt to host defense immunity, inflammation, tissue development, and substance metabolism, and interact with the host to form symbiotic networks $(54,55)$. Esophageal carcinogenesis has a complicated course that is affected by genetic and environmental factors (56). Some of these carcinogenesisrelated mechanisms, including inflammation and immune regulation, microbial components, and the production of genotoxins, are closely linked to the gut microbiota.

\section{Inflammation and Immune Regulation}

Several studies have reported that the host immune system play a vital role in the carcinogenesis of gastrointestinal cancer (57). Gut microbiota could not only affect the local immunity of the digestive tract mucosa but also initiate the systemic immune response through whole-body immune cells (58). Furthermore, microflora and immune disorders may lead to inflammation, and consistent chronic release of inflammatory mediators is usually a initial contributing factor of cancer (59). The important influence of the microflora on the host immune system derives from the study of aseptic animals, which lack intestinal microflora $(54,60)$. Lee et al. found defects in the development of the immune system of these mice, which was characterized by a decrease in the number of CD4+ T cells and a corresponding decrease in the expression of Toll-like receptors (TLRs) and MHC II in their intestinal epithelial cells (54, 60). Therefore, normal microorganisms may play an important role in the development of immunity. On the other hand, when the balance between the microflora and the body's immune system is disrupted, the immune system's response to prevent bacterial invasion may trigger tumor growth (17). For example, Boursi et al. found that disorders of the microflora and the immune system caused by penicillin were associated with EC or precancerous lesions (61).

Several studies have suggested possible mechanisms by which microflora interact with the body's immune system and signaling pathways to cause cancer. The imbalance between human microflora and the immune system may change the composition of normal microflora in the esophagus and form a microbial-related molecular model (59), including TLR and nucleotide-binding oligomeric domain (NOD)-like receptors $(62,63)$. Pursuant activation of related receptors might result in the production and release of cytokines and chemokines involved in chronic inflammation to promote the occurrence and progress of cancer (64). For example, BE epithelial biopsies have high levels of proinflammatory cytokines, especially IL-1 $\beta$ (62). Moreover, some inflammatory factors released in the inflammatory condition (such as IL-6 and IL-23) increase the reaction between microorganisms and the host by promoting the inflammatory response, which causes cancer in the end (59). These findings confirm the potential role of inflammation and immune regulation in the development of EC (Figure 1).

\section{Microbial Components}

In addition to the effects of microbial disorders on the human immune system, bacterial material, such as bacterial cell wall components and DNA, can be used as ligands for some receptors on the esophageal epithelium. Esophageal type II microflora, dominated by Gram-negative bacteria, can produce abundant lipopolysaccharide (LPS) $(27,65)$. LPS can delay gastric emptying through cyclooxygenase $1 / 2$ or directly affect the function of the lower esophageal sphincter, and then increase intragastric pressure to promote the occurrence of GERD, leading to the development of EAC $(65,66)$. Interestingly, as a natural ligand of LPS, the expression of TLR4 increases in the esophageal epithelium of patients with BE and EAC (65). The activation of TLR4 receptor triggers the NF-kappa B pathway associated with inflammation-related carcinogenesis (67) and mediates the formation of early BE (68). Therefore, inflammation and malignant transformation of the esophagus may be accomplished through the activation of the LPS-TLR4NF- $\kappa B$ pathway $(65,69)$. In addition, Nadatani et al. found that after BE cells were treated with LPS, the expression of NOD-like receptor protein 3 (NLRP3), the activity of caspase-1, and the secretion of IL-1 $\beta$ and IL-18 increased, which they thought were due to the activation of reactive oxygen species (ROS) by LPS, and these promoted the development of cancer (70) (Figure 2).

Moreover, Baba et al. found that in ESCC tissues with Fusobacterium nucleatum, the number of specific chemokine (CCL20) genes increased, which indicated that F. nucleatum might promote the invasive behavior of esophageal tumors by motivating chemokines (such as CCL20) (71). It is well known that the vital roles of chemokines and their receptors in tumor development and progression in several types of cancers (72). As 


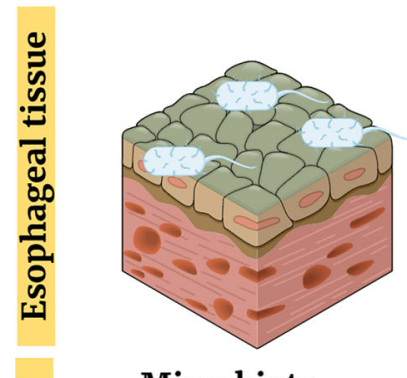

Microbiota banlance

Nomal physiological balance of the microflora and the immune system

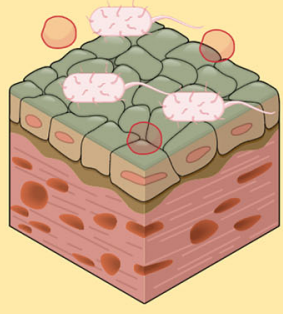

Microbiota imbanlance

Destruction of the physiological balance of the microflora and the immune system, contribute to the persistent chronic inflammation.

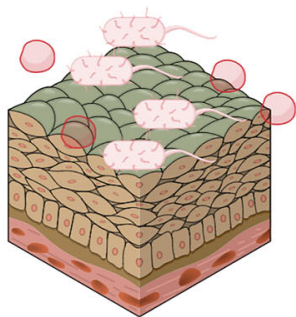

\section{Cancer or precancerous lesions}

The inflammatory cytokines activate the TLR and NOD-like receptor that may lead to carcinoma or precancerous lesions.

FIGURE 1 | Inflammation and immune regulation mechanism involved in the pathogenesis of EC. Factors (such as antibiotics) lead to the destruction of the physiological balance of the microflora and the immune system, which contribute to the persistent chronic inflammation. Moreover, the inflammatory cytokines activate the TLR and NOD-like receptor that may lead to carcinoma or precancerous lesions.

to their results, the most upregulated chemokine in F. nucleatum positive ESCC is CCL20. Remarkably, an increasing number of studies have recently drawn attention to the association of CCL20 and its receptor CCR6 in the oncogenesis of various types of cancers (73). For instance, Wang et al. have reported that CCL20 stimulation promoted cancer cell proliferation and migration in vitro (74).

\section{Production of Genotoxins}

Other than the components of the microbiota itself, some bacteria may directly produce genetic toxins or cancerpromoting metabolites that may cause genome damage and lead to EC. For example, cellular lethal swelling toxins secreted by Gram-negative bacteria may cause host DNA damage (75, 76), and further repair of DNA damage might lead to the development of EC. For instance, Gabriel et al. reported that the exposure of cultured mammalian epithelial cells to E. coli induced a DNA damage response followed by cell division with signs of incomplete DNA repair, leading to anaphase bridges and chromosome aberrations. Exposed cells exhibited a significant increase in gene mutation frequency and anchorage-independent colony formation, demonstrating the infection mutagenic and transforming potential (76).

In addition, cytotoxin-associated gene A (CagA) and vacuolating cytotoxin A produced by $H$. pylori can stimulate inflammation and lead to the development of cancer (77). CagA is a cancer-related protein, which could induce DNA damage through host-mediated upregulation production of $\operatorname{ROS}(78,79)$. Moreover, vacuolating cytotoxin A can change membrane permeability and lead to apoptosis rates increase (80). For instance, Li et al. found that CagA1-positive H. pylori can cause DNA breaks in esophageal epithelial cells, which can lead to atypical hyperplasia of esophageal squamous epithelial tissues and contribute to the carcinogenesis of ESCC. Further mechanism research found that $H$. pylori infection induces ROS in the cytoplasm, which promotes the DNA damage response (41) (Figure 3).

Generally, inflammation, the immune response, microbial components, and toxic products are the main mechanisms by which the gut microbiota promotes malignant transformation of the esophagus.

\section{USING THE MICROBIOTA AS BIOMARKERS}

One growing translational application of the digestive tract microbiota is to use them as biomarkers for screening and prognosis prediction. Accumulating evidence suggests that precise and convenient screening tests could notably decrease the global burden of EC (81). Moreover, several studies have reported the relationship between specific bacterial markers and clinical outcomes. In this section, we discuss microbiota-related biomarkers (mainly oral and esophageal microbiota) for EC screening and prognosis prediction (Table $\mathbf{1}$ ).

\section{Biomarkers for Screening}

It is necessary to identify precise biomarkers for screening early EC that can be cured with favorable clinical outcomes. Combining culture and non-culture methods, Blackett et al. compared the microbiota of a control group with those of patients with GERD, BE, and EAC. They found that the Campylobacter load in EAC was significantly lower than that 


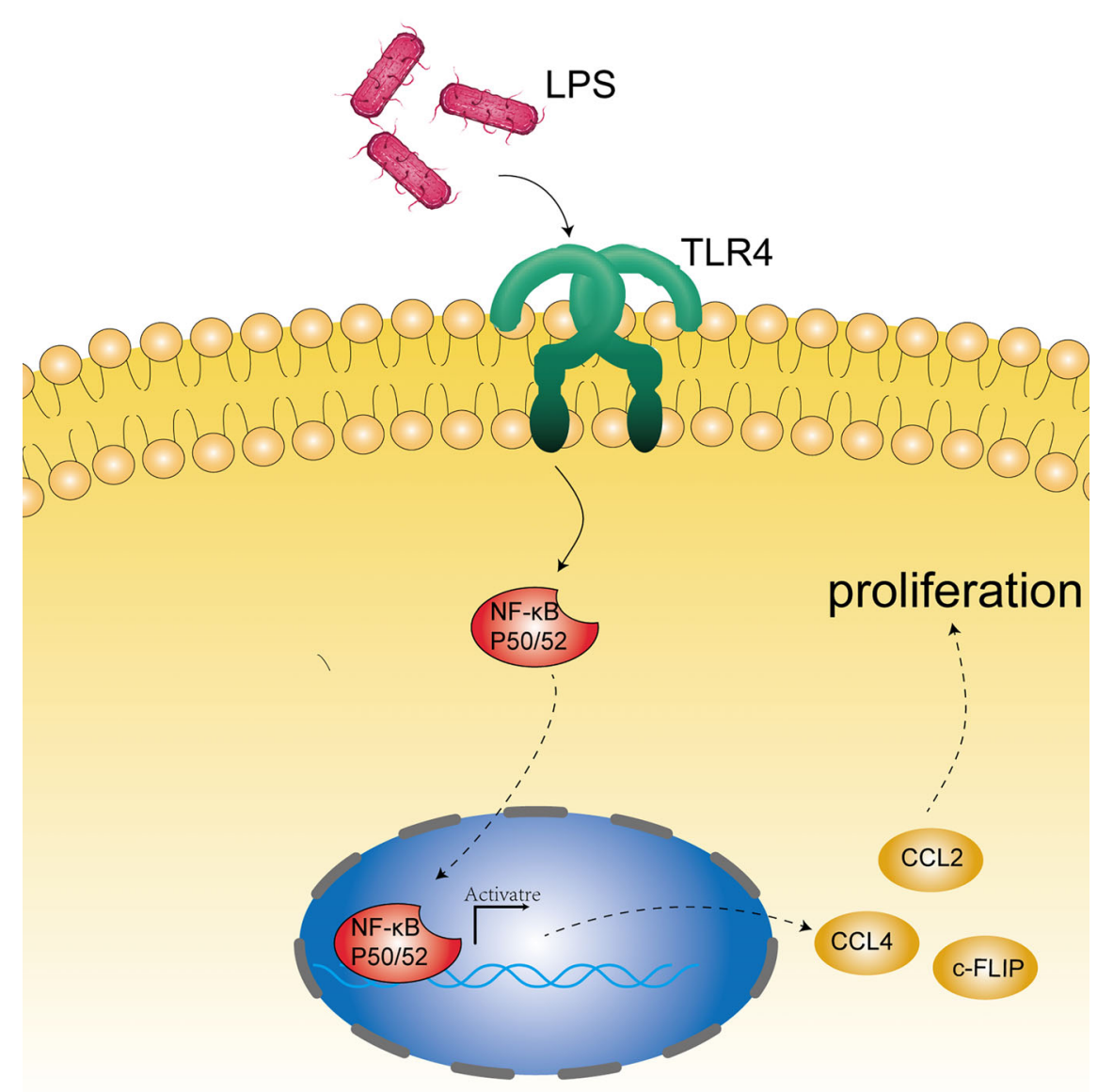

FIGURE 2 | Microbial component mechanism involved in the pathogenesis of EC. Bacterial constituents, such as bacterial cell wall components (mainly LPS) and DNA, may stimulate the receptors on the epithelium (such as TLR4) and activate the NF-kappa B pathway associated with carcinogenesis.

in GERD and BE $(82,86)$. In addition, the expression of carcinogenesis-related cytokines, such as IL-18, was higher in Campylobacter-colonized tissues $(82,86)$. Considering the potential effect of Campylobacter on human tumorigenesis (33), its role in the progression of EAC may be similar to that of H. pylori in gastric cancer. Consequently, it is reasonable to believe that Campylobacter may be a biomarker of EC. In addition, Elliott et al. found that Lactobacillus fermentum was enriched in EAC and the abundance of Lactobacillus was closely related to the development of EAC (36). Moreover, Zhou et al. reported a unique EAC microbiota containing a high abundance of Staphylococcus, Lactobacillus, Bifidobacterium, and Streptococcus. Their results suggested these microbiotas can be diagnosis biomarkers because of the close relationship between them and the appearance of EAC (87).

In addition to the microflora of the esophagus itself, oral microbiota serves as biomarkers for the early diagnosis of EC. The esophageal microbiota are largely affected by the oral microflora, which might be due to their close proximity anatomically, and the composition of the oral microbiota can provide some evidence of the progress of EC (53). Peters et al. found that the composition of oral microflora can reflect the potential risk of EAC, and according to their conclusion, Neisseria and S. pneumoniae/P. gingivalis were positively related to the presence of EAC/ESCC, respectively (48). Moreover, Chen et al. found that Prevos, Pseudomonas, and Streptococcus were more abundant in the saliva of patients with ESCC than in that of a normal group (30). Furthermore, a study published in 2018 constructed a model including the relative abundance of Lautropia, Streptococcus, and Bacteroidales in order to distinguish BE from controls, which has an area under the SUV curve of 0.94 (29).

\section{Biomarkers for Cancer Prognostication}

Apart from the potential capability of microbiota for EC diagnosis, combinations of microbial biomarkers and clinical outcomes of EC have allowed the use of microbiota as prognostic markers. 


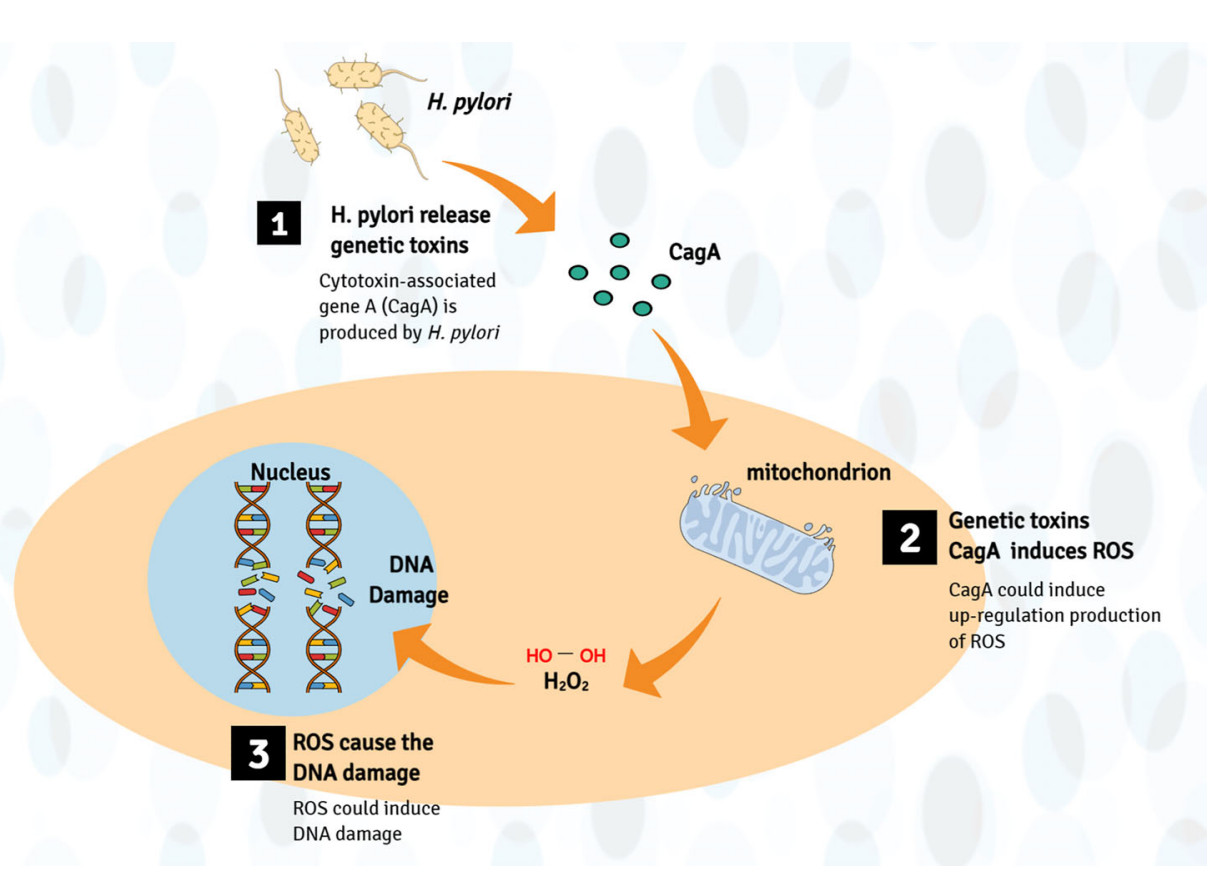

FIGURE 3 | Production of genotoxins mechanism involved in the pathogenesis of EC. Bacteria (such as H. pylori) may directly produce genetic toxins (mainly CagA) or cancer-promoting metabolites, which may cause genome damage by upregulating the production of ROS, leading to esophageal cancer (host DNA damage).

Among all the microorganic candidates, F. nucleatum seems a crucial prognostic marker. Yamamura et al. found that a high level of F. nucleatum in the tumor was associated with a larger tumor size, higher T stage, and higher TNM stage. In addition to being associated with EC staging, they also noted that the total load of F. nucleatum in tumor tissues of patients with recurrent EC was significantly higher than that of patients without EC recurrence (85). Moreover, the prognosis was significantly worse in patients with a high load of F. nucleatum (85). Furthermore, Gao et al. found that the load of $P$. gingivalis was positively associated with the progression and poor clinical prognosis of ESCC (28). Therefore, F. nucleatum and P. gingivalis are possible prognostic markers of ESCC.

It has also been reported that multiple microbiota could be combined to form a novel indicator of EC outcomes, and Liu et al. found that the T3-4 group of EC had an increased abundance of Streptococcus compared to T1-2. Likewise, the N staging showed no exception; they found that patients with lymph node metastasis had a higher abundance of Prevotella and Treponema compared to control subjects (84). They also investigated the impact of the microbiota on the long-term prognosis of patients undergoing EC surgery and reported that the abundance of the genera Prevotella and Streptococcus was inversely correlated with survival rates in ESCC patients, suggesting that a high abundance of these genera predicts poor prognosis. Therefore, they proposed a new index to estimate prognosis based on a combination of the Streptococcus and Prevotella load (84).

Collectively, the above studies show that the gut microbiota play a key role in the screening of early EC patients and are able to represent the clinical outcome of EC patients. These studies reveal the potential of microbiota to serve as specific biomarkers for screening and prognosis. More clinical trials are needed to accelerate the clinical application of basic experiments related to gut microbiota.

\section{MODULATING MICROBIOTA FOR ESOPHAGEAL CANCER PREVENTION}

Preventive measures are an attractive strategy for reducing the burden of EC. Extensive epidemiological studies have identified several risk factors for EC, including dietary patterns, obesity, and other lifestyle factors, which may be readily modifiable (88). In addition, antibiotics (89) and probiotics (90) have been studied in the context of EC prophylaxis. Here, we review the potential of these factors to reduce EC by regulating gut microbiota.

\section{Administration of Probiotics}

Probiotics are live microbes that benefit health by improving the gut microbiota after being administered (91). The first discovery of probiotics was made in 1905 (92); recently, the anticancer activities of these microorganisms and their potential immune mechanisms have aroused interest (93). For EC, several probiotics including Bifidobacterium and Lactobacillus spp. have shown anticancer activities in clinical studies, for example, Zhang et al. reported that fermented dairy foods, which are known to have probiotic content (such as 
TABLE 1 | Gut microbiota biomarkers for EC screening and prognostication.

Stud

Population(s)

Study sample size

Study Study

Main findings

Marker

period platform

type

Blackett et al. (82) British 37 GERD, 45 BE, 30 EAC,

2013 qPCR

The abundance of Campylobacter in esophageal adenocarcinoma was significantly lower than that in GERD

Diagnosis and 39 healthy controls

Chen et al. (30)

Chinese

87 ESCC and 85 healthy

2015 16S rRNA

and Barrett's esophagus.

Elliott et al. (36) British 19 EAC and 20 healthy

controls

Peter et al. (48) American

controls

$25 \mathrm{ESCC}$ and 50 healthy

controls

Snider et al. (29) American $32 \mathrm{BE}$ and 17 healthy

controls

Zhou et al.

Australian

6 EAC and 16 healthy

controls

Yang et al. (46) Chinese

18 ESCC and 11 healthy

controls

Deng et al. (83) Chinese

$23 \mathrm{EC}$ and 23 healthy

controls

Gao et al. (28) Chinses 100 ESCC

Liu et al. (84) Chinses 45 ESCC

Yamamura et al. (85) Japanese

551 ESCC

Li et al. (45)

Chinese

17 ESCC, 15 patients at 9-

12 months after ESCC

2017 16S rRNA Lactobacillus fermentum was enriched in esophageal adenocarcinoma.

Diagnosis

2017 16S rRNA Neisseria and Streptococcus pneumoniae/Porphyromonas gingivalis were positively related to the presence of Diagnosis EAC/ESCC, respectively

2018 16S rRNA A model including relative abundance of Lautropia, Streptococcus, and Bacteroidales in order to distinguish BE from controls with an area under the ROC curve of 0.94

2020 16S rRNA A high abundance of Staphylococcus, Lactobacillus, Bifidobacterium, and Streptococcus, which contribute towards dysregulated lactic acid-producing, consist of a distinct EAC microbiota.

2021 16S rRNA Employing decreased abundance of Bacteroidetes, Fusobacteria, and Spirochaetes into a microbial dysbiosis index showed that dysbiosis microbiota had an excellent capacity to discriminate between ESCC and normal subjects.

2021 16S rRNA ROC analysis revealed that Lachnospira, Bacteroides, Streptococcus, and Bifidobacterium achieved an area under the curve that was more than 0.85 , showing high accuracy in EC diagnosis

2016 GPCR Porphyromonas gingivalis was also positively correlated with the severity and poor clinical prognosis of ESCC. Prognosis

2018 16S rRNA The abundances of genera Prevotella and Streptococcus were inversely correlated with suvival rates in ESCC Prognosis patients

2019 gPCR High level of Fusobacterium nucleatum in the tumor was associated with larger tumor size, higher T stage, higher TNM stage, and worse prognoses.

radical esophagectomy and

Bacteroidetes and Pseudomonas were the key taxa contributing to the changes in the microbiome of the

ESCC and post-ESCC groups which were similar to the healthy control group. The monitoring of the

Bacteroidetes and Pseudomonas may be an essential method to predict the recurrence of the tumor.

Diagnosis

Diagnosis

Diagnosis

Diagnosis

Prognosis

Prognosis

healthy controls 
Bifidobacterium), intake decreased EC risk significantly (90). However, the specific mechanisms supporting probiotics to prevent EC are still lacking. More in vivo and in vitro studies are needed to help determine the role of probiotics in preventing EC.

\section{Exposure to Antibiotics}

It is well known that antibiotics play a crucial role in regulating the gut microbiota $(94,95)$. Exposure to antibiotics during treatment for infectious diseases may have dramatically changed the gut microbial compositions, which are related to differing EC risks.

Several studies have made progress in the effects of administrating antibiotics or not on EC risk in general. Conflicting results were reported with observational data showing an increase (61) or no change (89) in risk of EC after antibiotic consumption. In detail, Akinari et al. investigated whether alteration of microbiota using penicillin $G$ and streptomycin affects EAC development. As to their study, incidence rates of $\mathrm{BE}$ and $\mathrm{EAC}$ were no statistical difference between antibiotic and control groups, although the antibiotics group has a trend to reduced incidence of EAC and the esophageal microbiome was different between the two groups (89). Conversely, a study with 125,441 cases and 490,510 matched controls was analyzed by Ben et al. For gastrointestinal malignancies, the use of penicillin was associated with an elevated risk of esophageal, gastric, and pancreatic cancers (61). Therefore, studies with a higher quality of evidence need to be conducted to elucidate the preventive effect of antibiotic regulation of gut microbiota on $\mathrm{EC}$

\section{Dietary Interventions}

Diet is a significant determinant of gut microbes (96). People who ate different diets had significantly different gut microbiome compositions associated with different EC risks. Given that dietary interventions (particularly fiber and fat intake) can profoundly reshape our microbiota (97), interest in dietary interventions to influence the occurrence and progression of gut microbiota to prevent EC has been piqued.

That dietary fat intake can dramatically affect the composition of the gut microbiota has been proved in mouse models (98). Natasha et al. reported that BE model mice fed an HFD developed esophageal dysplasia and tumors more rapidly than mice fed the control diet, which was associated with a shift in the gut microbiota. They observed similar differences in the microbiomes from patients with $\mathrm{BE}$ who progressed to EAC and those who did not develop into cancer (99). Besides, Jeffrey et al. demonstrate that chronic HFD alone induced esophageal inflammation and metaplasia via increased microbiota diversity (100).

In addition, studies on individual dietary composition have indicated that dietary fiber, which can be acquired from natural food products or supplemented as a prebiotic preparation, is an essential factor affecting gut microbial diversity (77). Prebiotics are compounds in foods that induce the growth or activity of beneficial microorganisms such as bacteria by altering the microbial composition of the gut microbiome (101). Dietary fiber intervention could increase Bifidobacterium and Lactobacillus spp.'s abundance and boost fecal butyrate concentration in humans (102) through microbial fermentation. Importantly, Nobel et al. collected esophageal samples from 47 ambulatory patients completed a validated food frequency questionnaire quantifying dietary fiber and fat intake to determine the association of the composition of the esophageal microbiome and fiber intake. Their results showed that increasing fiber intake was significantly associated with the increasing relative abundance of Firmicutes. Therefore, dietary fiber intake was an essential modifier of the esophageal microbiome, which has the potential to prevent esophageal diseases (103).

\section{Weight Reduction}

Obesity is an assured risk factor for EC (especially EAC), and there seemingly is a linear association between increased body mass index and EAC (104). In addition, multivariable analysis showed that systemic or central obesity was an independent risk factor for EAC (105). Obesity may cause EC through such mechanisms as insulin and insulin-like growth factor signaling, chronic inflammation, and adipokines (106). In addition, the gut microbiota has emerged as a novel mechanism that regulates systemic exposure to bacterial LPS, secondary to changes in intestinal permeability, leading to metabolic disorders, insulin resistance, and thereby promoting EC formation $(106,107)$.

Previous studies have reported that obesity is associated with reduced microbial diversity (108) and changes in gut microbiome composition (109). Thus, weight control in obese individuals can profoundly alter their gut microbiota (110). Obese individuals who continued to lose weight after bariatric surgery had a lower risk of developing obesity-related cancers than matched obese controls (111), although this study lacked the ability to assess the risk of specific cancer types. Therefore, studies with large sample sizes are urgently needed to specifically clarify whether weight reduction could affect the incidence of EC by altering the gut microbiota.

\section{HARNESSING MICROBIOTA FOR ESOPHAGEAL CANCER THERAPY-RELATED APPLICATIONS}

The composition of gut microbiota can be changed by antibiotics (112), prebiotics (113), probiotics (114), or microflora transplantation (115). The treatment of EC by regulating the microflora may become a novel treatment method. Narrow-band antibiotics can selectively remove or inhibit harmful components of the human gut microbiota. Prebiotics can promote the proliferation of beneficial microorganisms. Probiotics can introduce beneficial microbial components that are not present in human hosts. Fecal microflora transplantation may target microflora to treat cancer. However, there are still no reported cases of intestinal microflora use to treat EC directly. Therefore, most applications of gut microbiota are to strengthen the efficacy of neoadjuvant or adjuvant treatment and to prevent complications caused by therapeutic methods. 


\section{Harnessing Microbiota to Enhance the Efficacy of Treatment}

In addition to its roles in the direct prevention of EC, there is growing evidence that the gut microbiota could mediate the efficacy of chemotherapy and immunotherapy. This enables their use as biomarkers to predict treatment response and, at the same time, to be modulated to enhance cancer treatment efficacy.

A growing body of evidence suggests that the gut microbiota can adjust the anticancer chemotherapeutic effects by mechanisms of microbial immunomodulation, metabolism, and translocation (116). For instance, it has been reported that the microbiota can improve the efficacy of oxaliplatin because the gut microbiota stimulate immune cells to produce ROS, which enhance the DNA damage caused by oxaliplatin, leading to cell necrosis (55). However, a study that included 30 patients assigned to administrate synbiotics (combination of probiotic and prebiotic) in the course of chemotherapy and 31 control subjects was reported by Masaaki et al. The clinical response rate was 60 and 52\% after chemotherapy to synbiotics and control group, respectively. No significant difference but a tendency that synbiotics could promote the effect of chemotherapy was observed in the response rates of the two groups. Since the sample size of the present study was small, larger-scale studies need to demonstrate whether the synbiotics could improve chemotherapy in the future (12). Furthermore, Cheung et al. investigated the association of gut microbiota and EC treatment efficacy by FMT. As to their results, healthy mouse stools did not significantly affect anti-EC medicinal herb Andrographis paniculata (AP) efficacy. However, the antibiotic treatment reduced the efficacy of AP from 89.5 to $46.8 \%$ in microbiota-intact and microbiota-depleted mice, respectively. These findings demonstrate that the efficacy of AP for EC depends, at least partly, on the commensal gut microbiota (47). In addition, Yamamura et al. studied whether the high load of $F$. nucleatum in the tumor of ESCC patients is related to the efficacy of neoadjuvant chemotherapy. All patients were pathologically evaluated with imaging data provided by CT scans, metabolic response rates determined by the maximum standardized uptake value from $\mathrm{PET} / \mathrm{CT}$ imaging, and tumor regression grade analysis. The results showed that patients with high loads of $F$. nucleatum in tumors seemed to be more resistant to neoadjuvant chemotherapy treatment (85); therefore, the use of narrowspectrum antibiotics to eradicate $F$. nucleatum might increase the effectiveness of neoadjuvant chemotherapy in ESCC patients.

Considering that the effect of microbiota on EC plays a role in the immune system, it is reasonable to presume that microflora may also affect the response of their hosts to immunotherapy. Immunotherapy is a valid method for treating many types of cancer and has become a complementary method after surgical treatment, radiotherapy, and chemotherapy. Immune checkpoint inhibitors can reactivate tumor-reactive $\mathrm{T}$ cells to induce antitumor immune responses (117). The normal gut microbiota are necessary for an effective immune response caused by programmed cell death 1 or programmed cell death ligand 1 (118-121) and cytotoxic T lymphocyte-associated antigen-4 (122). Previous studies have reported that Akkermansia muciniphila
(120), Eubacterium limosum (123), and Alistipes shahii (55) were positively associated with immunotherapeutic effects. Importantly, a study has reported that the use of antibiotics can weaken the effect of immunotherapy by $\mathrm{CpG}$ oligonucleotides in mice with subcutaneous tumors such as EC (55).

\section{Harnessing Microbiota to Prevent Therapy-Related Complications}

Interestingly, in addition to the prevention and treatment of EC itself, gut microbiota is also related to complications in the treatment process, which fully demonstrates the close relationship between gut microbiota and EC. For instance, Tanaka et al. found that the duration of the systemic inflammatory response syndrome after esophagectomy was significantly shorter in the synbiotic group than in the control group (51). In addition, Giamarellos-Bourboulis found that administering synbiotics can reduce endotoxin, white blood cell count, C-reactive protein level, and the incidence of septic complications $(124,125)$ after EC surgery. This may be because the modification of intestinal microflora with synbiotics can weaken the overgrowth of bacteria in the intestine and reduce the translocation of bacteria to distant organs $(126,127)$. Moreover, Okada et al. reported that Bifidobacterium can reduce the expression of proinflammatory cytokines, which may be related to the inhibition of Ikappa B- $\alpha$ phosphorylation. These results suggest that rebuilding beneficial gut microbiota may reduce excessive inflammatory responses through direct immunomodulatory effects (128).

In addition to preventing complications from surgery, modulating the microbiota can also reduce the incidence of complications caused by esophageal chemotherapy. For instance, Masaaki et al. found that synbiotics distinctly weakened the severity of lymphopenia after EC chemotherapy (12), because synbiotics sustained the gut microbiota and the relatively low $\mathrm{pH}$, which improved the nutritional status of colonocytes. Most importantly, relevant studies have revealed that the gut microbiota has potential application either for enhancing the efficacy of treatment or for preventing complications. More research is needed to assess the potential of these corresponding microbiota for clinical applications in the context of EC.

\section{CURRENT CHALLENGES AND FUTURE PROSPECTS}

Extensive studies have identified the importance of EC microbiota, which interact closely with host esophageal epithelial cells and play an important role in the development of EC and in the elucidation of the mechanisms of carcinogenesis. Hence, there are unprecedented opportunities to find new ways of diagnosing and treating EC, and there is a scientific challenge in identifying different biomarkers. In the past, oral and esophageal microflora were mainly studied as predictive markers of EC $(30,48)$. However, in addition to the oral cavity and esophagus, convenient samples can also be obtained from feces; thus, finding predictive markers of EC in feces requires further research. It is hoped that in the near future, relevant microflora will be found, and large randomized controlled trials will 


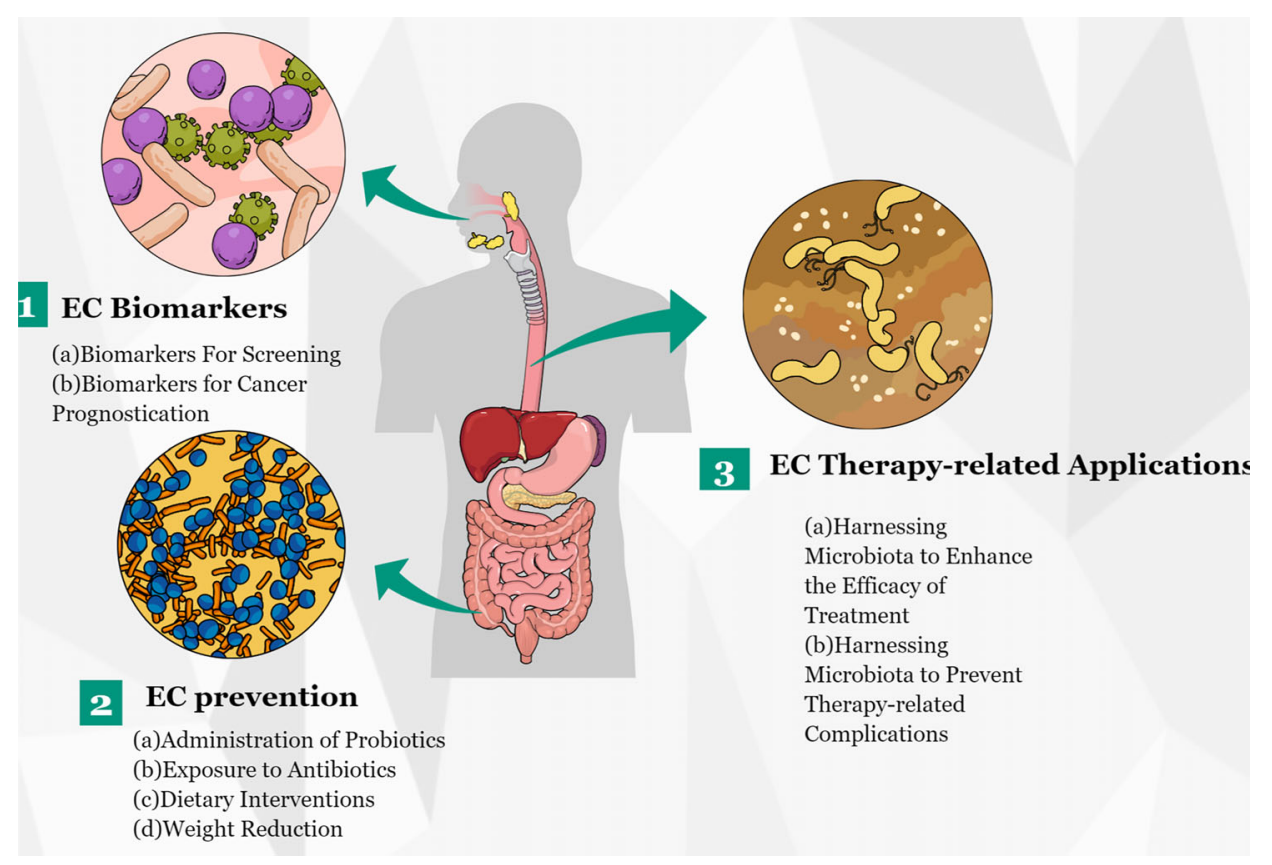

FIGURE 4 | Potential clinical applications related to gut microbiota in EC. Several potential clinical applications for harnessing the gut microbiota in EC are depicted and include the development of screening, prognostic, and predictive biomarkers, and microbiota modulation for EC prevention and treatment.

be conducted to prove their efficacy. In addition, the success of fecal microflora transplantation in treating recurrent Clostridium difficile has stimulated considerable interest in manipulating gut microbiota, but there is currently little consensus on the best intervention approach (129). Therefore, efforts need to be made to find the best way to intervene and manipulate the gut microbiome. In addition to fecal microflora transplantation, several advantageous microbial species can be administered as probiotics, and the direct clinical benefits and associated microbial benefits in EC also remain to be determined. In addition, studies on the influence of intestinal microorganisms on the prognosis of EC mainly focus on the prediction of EC stage, and it is important to establish an intestinal flora model to predict the sensitivity of radiotherapy, chemotherapy, and immunotherapy for EC and the incidence of postoperative complications. The use of probiotics to reduce complications after esophagectomy and chemoradiotherapy requires additional supporting data from the trial, and efforts are needed to further understand the carcinogenic effects of gut microbes and the mechanisms that regulate tumor response to treatment. With developments in this rapidly evolving field, the microbiota will be an important part of cancer prevention and treatment.

\section{CONCLUSION}

In the last several years, a growing number of studies has revealed the vital role of microbiota in EC and have suggested that an imbalance in gut microbiota might lead to esophageal tumorigenesis. In addition, several research groups have conducted functional studies to verify the role of individual microbiota in carcinogenesis. In summary, these reported results offer an unprecedented opportunity for the translation of microbiota discoveries to clinical applications (Figure 4). With the progress of research in EC metagenomics and metabolomics, microbiota discoveries will potentially enrich treatment modalities for EC in the near future.

\section{AUTHOR CONTRIBUTIONS}

YY conceptualized the study, revised the manuscript, and supervised the study. JZ and SS conceptualized the study, drafted the manuscript, and made the figures. SL, XX, YSY, $\mathrm{CM}, \mathrm{LC}, \mathrm{XZ}$ and $\mathrm{YZ}$ collected the literature and revised the manuscript. All authors contributed to the article and approved the submitted version.

\section{FUNDING}

This study was supported by the National Natural Science Foundation of China (Grant No. 81970481) and 1.3.5 project for disciplines of excellence, West China Hospital, Sichuan University (Grant Nos. 2020HXFH047 and 20HXJS005). 


\section{REFERENCES}

1. Smyth EC, Lagergren J, Fitzgerald RC, Lordick F, Shah MA, Lagergren P, et al. Oesophageal Cancer. Nat Rev Dis Primers (2017) 3:17048. doi: 10.1038/ nrdp.2017.48

2. Thrift AP. Global Burden and Epidemiology of Barrett Oesophagus and Oesophageal Cancer. Nat Rev Gastroenterol Hepatol (2021) 18(6):432-43. doi: 10.1038/s41575-021-00419-3

3. Sung H, Ferlay J, Siegel RL, Laversanne M, Soerjomataram I, Jemal A, et al. Global Cancer Statistics 2020: GLOBOCAN Estimates of Incidence and Mortality Worldwide for 36 Cancers in 185 Countries. CA: Cancer J Clin (2021) 71(3):209-49. doi: 10.3322/caac.21660

4. Gupta B, Kumar N. Worldwide Incidence, Mortality and Time Trends for Cancer of the Oesophagus. Eur J Cancer Prev (2017) 26(2):107-18. doi: 10.1097/CEJ.0000000000000249

5. Lagergren J, Smyth E, Cunningham D, Lagergren P. Oesophageal Cancer. Lancet (2017) 390(10110):2383-96. doi: 10.1016/S0140-6736(17)31462-9

6. Lichtenstein P, Holm NV, Verkasalo PK, Iliadou A, Kaprio J, Koskenvuo M, et al. Environmental and Heritable Factors in the Causation of CancerAnalyses of Cohorts of Twins From Sweden, Denmark, and Finland. N Engl $J$ Med (2000) 343(2):78-85. doi: 10.1056/NEJM200007133430201

7. Abnet CC, Arnold M, Wei WQ. Epidemiology of Esophageal Squamous Cell Carcinoma. Gastroenterology (2018) 154(2):360-73. doi: 10.1053/ j.gastro.2017.08.023

8. Coleman HG, Xie SH, Lagergren J. The Epidemiology of Esophageal Adenocarcinoma. Gastroenterology (2018) 154(2):390-405. doi: 10.1053/ j.gastro.2017.07.046

9. Kamangar F, Freedman ND. Hot Tea and Esophageal Cancer. Ann Internal Med (2018) 168(7):519-20. doi: 10.7326/M17-3370

10. Moszak M, Szulinska M, Bogdanski P. You Are What You Eat-The Relationship Between Diet, Microbiota, and Metabolic Disorders-A Review. Nutrients (2020) 12(4):1096. doi: 10.3390/nu12041096

11. Zhao Q, Yang T, Yan Y, Zhang Y, Li Z, Wang Y, et al. Alterations of Oral Microbiota in Chinese Patients With Esophageal Cancer. Front Cell Infect Microbiol (2020) 10:541144. doi: 10.3389/fcimb.2020.541144

12. Motoori M, Yano M, Miyata H, Sugimura K, Saito T, Omori T, et al. Randomized Study of the Effect of Synbiotics During Neoadjuvant Chemotherapy on Adverse Events in Esophageal Cancer Patients. Clin Nutr (Edinburgh Scotland) (2017) 36(1):93-9. doi: 10.1016/ j.clnu.2015.11.008

13. Yu Y, Gao F, Chen X, Zheng S, Zhang J. Changes in the Distal Esophageal Microbiota in Chinese Patients With Reflux Esophagitis. J Digestive Dis (2019) 20(1):18-24. doi: 10.1111/1751-2980.12692

14. Plummer M, de Martel C, Vignat J, Ferlay J, Bray F, Franceschi S. Global Burden of Cancers Attributable to Infections in 2012: A Synthetic Analysis. Lancet Global Health (2016) 4(9):e609-16. doi: 10.1016/S2214-109X(16)30143-7

15. Liu Y, Yang K, Jia Y, Shi J, Tong Z, Fang D, et al. Gut Microbiome Alterations in High-Fat-Diet-Fed Mice are Associated With Antibiotic Tolerance. Nat Microbiol (2021) 6(7):874-84. doi: 10.1038/s41564-02100912-0

16. Hanus M, Parada-Venegas D, Landskron G, Wielandt AM, Hurtado C, Alvarez K, et al. Immune System, Microbiota, and Microbial Metabolites: The Unresolved Triad in Colorectal Cancer Microenvironment. Front Immunol (2021) 12:612826. doi: 10.3389/fimmu.2021.612826

17. Russo E, Taddei A, Ringressi MN, Ricci F, Amedei A. The Interplay Between the Microbiome and the Adaptive Immune Response in Cancer Development. Ther Adv Gastroenterol (2016) 9(4):594-605. doi: 10.1177/ $1756283 \mathrm{X} 16635082$

18. Mannell A, Plant M, Frolich J. The Microflora of the Oesophagus. Ann R Coll Surgeons Engl (1983) 65(3):152-4.

19. Yatera K, Noguchi S, Mukae H. Perspective on the Clone Library Method for Infectious Diseases. Respir Invest (2021) S2212-5345(21):00123-4. doi: $10.1016 /$ j.resinv.2021.07.003

20. Kurokawa K, Itoh T, Kuwahara T, Oshima K, Toh H, Toyoda A, et al. Comparative Metagenomics Revealed Commonly Enriched Gene Sets in Human Gut Microbiomes. DNA Res An Int J Rapid Publ Rep Genes Genomes (2007) 14(4):169-81. doi: 10.1093/dnares/dsm018
21. Hugenholtz P, Goebel BM, Pace NR. Impact of Culture-Independent Studies on the Emerging Phylogenetic View of Bacterial Diversity. J Bacteriol (1998) 180(18):4765-74. doi: 10.1128/jb.180.18.4765-4774.1998

22. Case RJ, Boucher Y, Dahllof I, Holmstrom C, Doolittle WF, Kjelleberg S. Use of $16 \mathrm{~S}$ rRNA and rpoB Genes as Molecular Markers for Microbial Ecology Studies. Appl Environ Microbiol (2007) 73(1):278-88. doi: 10.1128/ AEM.01177-06

23. Caporaso JG, Lauber CL, Walters WA, Berg-Lyons D, Lozupone CA, Turnbaugh PJ, et al. Global Patterns of 16S rRNA Diversity at a Depth of Millions of Sequences Per Sample. Proc Natl Acad Sci U S A (2011) 108 Suppl 1:4516-22. doi: 10.1073/pnas.1000080107

24. Clarridge JE3rd. Impact of $16 \mathrm{~S}$ rRNA Gene Sequence Analysis for Identification of Bacteria on Clinical Microbiology and Infectious Diseases. Clin Microbiol Rev (2004) 17(4):840-62. doi: 10.1128/ CMR.17.4.840-862.2004

25. Breitbart M, Salamon P, Andresen B, Mahaffy JM, Segall AM, Mead D, et al. Genomic Analysis of Uncultured Marine Viral Communities. Proc Natl Acad Sci U S A (2002) 99(22):14250-5. doi: 10.1073/pnas.202488399

26. Anderson S. Shotgun DNA Sequencing Using Cloned DNase I-Generated Fragments. Nucleic Acids Res (1981) 9(13):3015-27. doi: 10.1093/nar/ 9.13.3015

27. Yang L, Lu X, Nossa CW, Francois F, Peek RM, Pei Z. Inflammation and Intestinal Metaplasia of the Distal Esophagus are Associated With Alterations in the Microbiome. Gastroenterology (2009) 137(2):588-97. doi: 10.1053/j.gastro.2009.04.046

28. Gao S, Li S, Ma Z, Liang S, Shan T, Zhang M, et al. Presence of Porphyromonas Gingivalis in Esophagus and Its Association With the Clinicopathological Characteristics and Survival in Patients With Esophageal Cancer. Infect Agents Cancer (2016) 11:3. doi: 10.1186/s13027016-0049-x

29. Snider EJ, Compres G, Freedberg DE, Giddins MJ, Khiabanian H, Lightdale CJ, et al. Barrett's Esophagus is Associated With a Distinct Oral Microbiome. Clin Trans Gastroenterol (2018) 9(3):135. doi: 10.1038/s41424-018-0005-8

30. Chen X, Winckler B, Lu M, Cheng H, Yuan Z, Yang Y, et al. Oral Microbiota and Risk for Esophageal Squamous Cell Carcinoma in a High-Risk Area of China. PLoS One (2015) 10(12):e0143603. doi: 10.1371/journal. pone. 0143603

31. Wheeler JB, Reed CE. Epidemiology of Esophageal Cancer. Surg Clinics North Am (2012) 92(5):1077-87. doi: 10.1016/j.suc.2012.07.008

32. Rubenstein JH, Taylor JB. Meta-Analysis: The Association of Oesophageal Adenocarcinoma With Symptoms of Gastro-Oesophageal Reflux. Alimentary Pharmacol Ther (2010) 32(10):1222-7. doi: 10.1111/j.13652036.2010.04471.x

33. Macfarlane S, Furrie E, Macfarlane GT, Dillon JF. Microbial Colonization of the Upper Gastrointestinal Tract in Patients With Barrett's Esophagus. Clin Infect Dis (2007) 45(1):29-38. doi: 10.1086/518578

34. Liu N, Ando T, Ishiguro K, Maeda O, Watanabe O, Funasaka K, et al. Characterization of Bacterial Biota in the Distal Esophagus of Japanese Patients With Reflux Esophagitis and Barrett's Esophagus. BMC Infect Dis (2013) 13:130. doi: 10.1186/1471-2334-13-130

35. Yang L, Chaudhary N, Baghdadi J, Pei Z. Microbiome in Reflux Disorders and Esophageal Adenocarcinoma. Cancer J (2014) 20(3):207-10. doi: 10.1097/PPO.0000000000000044

36. Elliott DRF, Walker AW, O'Donovan M, Parkhill J, Fitzgerald RC. A nonEndoscopic Device to Sample the Oesophageal Microbiota: A Case-Control Study. Lancet Gastroenterol Hepatol (2017) 2(1):32-42. doi: 10.1016/S24681253(16)30086-3

37. Snider EJ, Compres G, Freedberg DE, Khiabanian H, Nobel YR, Stump S, et al. Alterations to the Esophageal Microbiome Associated With Progression From Barrett's Esophagus to Esophageal Adenocarcinoma. Cancer Epidemiol Biomarkers Prev Publ Am Assoc Cancer Res Cosponsored By Am Soc Prev Oncol (2019) 28(10):1687-93. doi: 10.1158/1055-9965.EPI19-0008

38. Lopetuso LR, Severgnini M, Pecere S, Ponziani FR, Boskoski I, Larghi A, et al. Esophageal Microbiome Signature in Patients With Barrett's Esophagus and Esophageal Adenocarcinoma. PLoS One (2020) 15(5):e0231789. doi: 10.1371/journal.pone.0231789 
39. Thrumurthy SG, Chaudry MA, Thrumurthy SSD, Mughal M. Oesophageal Cancer: Risks, Prevention, and Diagnosis. BMJ (2019) 366:14373. doi: $10.1136 / \mathrm{bmj} .14373$

40. Yu G, Gail MH, Shi J, Klepac-Ceraj V, Paster BJ, Dye BA, et al. Association Between Upper Digestive Tract Microbiota and Cancer-Predisposing States in the Esophagus and Stomach. Cancer Epidemiol Biomarkers Prev (2014) 23 (5):735-41. doi: 10.1158/1055-9965.EPI-13-0855

41. Li WS, Tian DP, Guan XY, Yun H, Wang HT, Xiao Y, et al. Esophageal Intraepithelial Invasion of Helicobacter Pylori Correlates With Atypical Hyperplasia. Int J Cancer (2014) 134(11):2626-32. doi: 10.1002/ijc.28588

42. Shao D, Vogtmann E, Liu A, Qin J, Chen W, Abnet CC, et al. Microbial Characterization of Esophageal Squamous Cell Carcinoma and Gastric Cardia Adenocarcinoma From a High-Risk Region of China. Cancer (2019) 125(22):3993-4002. doi: 10.1002/cncr.32403

43. Nieuwdorp M, Gilijamse PW, Pai N, Kaplan LM. Role of the Microbiome in Energy Regulation and Metabolism. Gastroenterology (2014) 146(6):152533. doi: $10.1053 /$ j.gastro.2014.02.008

44. Cani PD. Gut Cell Metabolism Shapes the Microbiome. Science (2017) 357 (6351):548-9. doi: 10.1126/science.aao2202

45. Li D, He R, Hou G, Ming W, Fan T, Chen L, et al. Characterization of the Esophageal Microbiota and Prediction of the Metabolic Pathways Involved in Esophageal Cancer. Front Cell Infect Microbiol (2020) 10:268. doi: $10.3389 /$ fcimb. 2020.00268

46. Yang W, Chen CH, Jia M, Xing X, Gao L, Tsai HT, et al. Tumor-Associated Microbiota in Esophageal Squamous Cell Carcinoma. Front Cell Dev Biol (2021) 9:641270. doi: 10.3389/fcell.2021.641270

47. Cheung MK, Yue GGL, Tsui KY, Gomes AJ, Kwan HS, Chiu PWY, et al. Discovery of an Interplay Between the Gut Microbiota and Esophageal Squamous Cell Carcinoma in Mice. Am J Cancer Res (2020) 10(8):2409-27.

48. Peters BA, Wu J, Pei Z, Yang L, Purdue MP, Freedman ND, et al. Oral Microbiome Composition Reflects Prospective Risk for Esophageal Cancers. Cancer Res (2017) 77(23):6777-87. doi: 10.1158/0008-5472.CAN-17-1296

49. Wang Q, Rao Y, Guo X, Liu N, Liu S, Wen P, et al. Oral Microbiome in Patients With Oesophageal Squamous Cell Carcinoma. Sci Rep (2019) 9 (1):19055. doi: 10.1038/s41598-019-55667-w

50. Kageyama S, Takeshita T, Takeuchi K, Asakawa M, Matsumi R, Furuta M, et al. Characteristics of the Salivary Microbiota in Patients With Various Digestive Tract Cancers. Front Microbiol (2019) 10:1780. doi: 10.3389/ fmicb.2019.01780

51. Tanaka K, Yano M, Motoori M, Kishi K, Miyashiro I, Ohue M, et al. Impact of Perioperative Administration of Synbiotics in Patients With Esophageal Cancer Undergoing Esophagectomy: A Prospective Randomized Controlled Trial. Surgery (2012) 152(5):832-42. doi: 10.1016/j.surg.2012.02.021

52. Li J, Jia H, Cai X, Zhong H, Feng Q, Sunagawa S, et al. An Integrated Catalog of Reference Genes in the Human Gut Microbiome. Nat Biotechnol (2014) 32(8):834-41. doi: 10.1038/nbt.2942

53. Gorkiewicz G, Moschen A. Gut Microbiome: A New Player in Gastrointestinal Disease. Virchows Arch (2018) 472(1):159-72. doi: 10.1007/s00428-017-2277-x

54. Lee YK, Mazmanian SK. Has the Microbiota Played a Critical Role in the Evolution of the Adaptive Immune System? Science (2010) 330(6012):176873. doi: $10.1126 /$ science. 1195568

55. Iida N, Dzutsev A, Stewart CA, Smith L, Bouladoux N, Weingarten RA, et al. Commensal Bacteria Control Cancer Response to Therapy by Modulating the Tumor Microenvironment. Science (2013) 342(6161):967-70. doi: $10.1126 /$ science. 1240527

56. Baiu I, Backhus L. Esophageal Cancer Surgery. JAMA (2020) 324(15):1580. doi: 10.1001/jama.2020.2101

57. Tilg H, Adolph TE, Gerner RR, Moschen AR. The Intestinal Microbiota in Colorectal Cancer. Cancer Cell (2018) 33(6):954-64. doi: 10.1016/ j.ccell.2018.03.004

58. Gopalakrishnan V, Helmink BA, Spencer CN, Reuben A, Wargo JA. The Influence of the Gut Microbiome on Cancer, Immunity, and Cancer Immunotherapy. Cancer Cell (2018) 33(4):570-80. doi: 10.1016/ j.ccell.2018.03.015

59. Schwabe RF, Jobin C. The Microbiome and Cancer. Nat Rev Cancer (2013) 13(11):800-12. doi: 10.1038/nrc3610
60. Tomkovich S, Jobin C. Microbiota and Host Immune Responses: A LoveHate Relationship. Immunology (2016) 147(1):1-10. doi: 10.1111/ imm. 12538

61. Boursi B, Mamtani R, Haynes K, Yang YX. Recurrent Antibiotic Exposure may Promote Cancer Formation-Another Step in Understanding the Role of the Human Microbiota? Eur J Cancer (2015) 51(17):2655-64. doi: 10.1016/ j.ejca.2015.08.015

62. Verbeek RE, Siersema PD, Vleggaar FP, Ten Kate FJ, Posthuma G, Souza RF, et al. Toll-Like Receptor 2 Signalling and the Lysosomal Machinery in Barrett's Esophagus. J Gastrointestinal Liver Dis JGLD (2016) 25(3):273-82. doi: 10.15403/jgld.2014.1121.253.rc2

63. Kinnebrew MA, Pamer EG. Innate Immune Signaling in Defense Against Intestinal Microbes. Immunol Rev (2012) 245(1):113-31. doi: 10.1111/ j.1600-065X.2011.01081.x

64. Grivennikov SI, Wang K, Mucida D, Stewart CA, Schnabl B, Jauch D, et al. Adenoma-Linked Barrier Defects and Microbial Products Drive IL-23/IL17-Mediated Tumour Growth. Nature (2012) 491(7423):254-8. doi: 10.1038/nature 11465

65. Yang L, Francois F, Pei Z. Molecular Pathways: Pathogenesis and Clinical Implications of Microbiome Alteration in Esophagitis and Barrett Esophagus. Clin Cancer Res (2012) 18(8):2138-44. doi: 10.1158/10780432.CCR-11-0934

66. Calatayud S, Garcia-Zaragoza E, Hernandez C, Quintana E, Felipo V, Esplugues JV, et al. Downregulation of nNOS and Synthesis of PGs Associated With Endotoxin-Induced Delay in Gastric Emptying. Am J Physiol Gastrointestinal Liver Physiol (2002) 283(6):G1360-7. doi: 10.1152/ ajpgi.00168.2002

67. Maeda S, Omata M. Inflammation and Cancer: Role of Nuclear factorkappaB Activation. Cancer Sci (2008) 99(5):836-42. doi: 10.1111/j.13497006.2008.00763.x

68. Souza RF, Krishnan K, Spechler SJ. Acid, Bile, and CDX: The ABCs of Making Barrett's Metaplasia. Am J Physiol Gastrointestinal Liver Physiol (2008) 295(2):G211-8. doi: 10.1152/ajpgi.90250.2008

69. Neto AG, Whitaker A, Pei Z. Microbiome and Potential Targets for Chemoprevention of Esophageal Adenocarcinoma. Semin Oncol (2016) 43 (1):86-96. doi: 10.1053/j.seminoncol.2015.09.005

70. Nadatani Y, Huo X, Zhang X, Yu C, Cheng E, Zhang Q, et al. NOD-Like Receptor Protein 3 Inflammasome Priming and Activation in Barrett's Epithelial Cells. Cell Mol Gastroenterol Hepatol (2016) 2(4):439-53. doi: 10.1016/j.jcmgh.2016.03.006

71. Yamamura K, Baba Y, Nakagawa S, Mima K, Miyake K, Nakamura K, et al. Human Microbiome Fusobacterium Nucleatum in Esophageal Cancer Tissue Is Associated With Prognosis. Clin Cancer Res (2016) 22(22):557481. doi: 10.1158/1078-0432.Ccr-16-1786

72. Verbeke H, Geboes K, Van Damme J, Struyf S. The Role of CXC Chemokines in the Transition of Chronic Inflammation to Esophageal and Gastric Cancer. Biochim Biophys Acta (2012) 1825(1):117-29. doi: 10.1016/j.bbcan.2011.10.008

73. Nandi B, Pai C, Huang Q, Prabhala RH, Munshi NC, Gold JS. CCR6, the Sole Receptor for the Chemokine CCL20, Promotes Spontaneous Intestinal Tumorigenesis. PLoS One (2014) 9(5):e97566. doi: 10.1371/journal. pone.0097566

74. Wang B, Shi L, Sun X, Wang L, Wang X, Chen C. Production of CCL20 From Lung Cancer Cells Induces the Cell Migration and Proliferation Through PI3K Pathway. J Cell Mol Med (2016) 20(5):920-9. doi: 10.1111/ jcmm. 12781

75. Arthur JC, Perez-Chanona E, Muhlbauer M, Tomkovich S, Uronis JM, Fan TJ, et al. Intestinal Inflammation Targets Cancer-Inducing Activity of the Microbiota. Science (2012) 338(6103):120-3. doi: 10.1126/science.1224820

76. Cuevas-Ramos G, Petit CR, Marcq I, Boury M, Oswald E, Nougayrede JP. Escherichia Coli Induces DNA Damage In Vivo and Triggers Genomic Instability in Mammalian Cells. Proc Natl Acad Sci U S A (2010) 107 (25):11537-42. doi: 10.1073/pnas.1001261107

77. Plottel CS, Blaser MJ. Microbiome and Malignancy. Cell Host Microbe (2011) 10(4):324-35. doi: 10.1016/j.chom.2011.10.003

78. Tsugawa H, Suzuki H, Saya H, Hatakeyama M, Hirayama T, Hirata K, et al. Reactive Oxygen Species-Induced Autophagic Degradation of Helicobacter 
Pylori CagA Is Specifically Suppressed in Cancer Stem-Like Cells. Cell Host Microbe (2012) 12(6):764-77. doi: 10.1016/j.chom.2012.10.014

79. Koeppel M, Garcia-Alcalde F, Glowinski F, Schlaermann P, Meyer TF. Helicobacter Pylori Infection Causes Characteristic DNA Damage Patterns in Human Cells. Cell Rep (2015) 11(11):1703-13. doi: 10.1016/ j.celrep.2015.05.030

80. Palframan SL, Kwok T, Gabriel K. Vacuolating Cytotoxin A (VacA), a Key Toxin for Helicobacter Pylori Pathogenesis. Front Cell Infect Microbiol (2012) 2:92. doi: 10.3389/fcimb.2012.00092

81. Gehrung M, Crispin-Ortuzar M, Berman AG, O'Donovan M, Fitzgerald RC, Markowetz F. Triage-Driven Diagnosis of Barrett's Esophagus for Early Detection of Esophageal Adenocarcinoma Using Deep Learning. Nat Med (2021) 27(5):833-41. doi: 10.1038/s41591-021-01287-9

82. Blackett KL, Siddhi SS, Cleary S, Steed H, Miller MH, Macfarlane S, et al. Oesophageal Bacterial Biofilm Changes in Gastro-Oesophageal Reflux Disease, Barrett's and Oesophageal Carcinoma: Association or Causality? Alimentary Pharmacol Ther (2013) 37(11):1084-92. doi: 10.1111/apt.12317

83. Deng Y, Tang D, Hou P, Shen W, Li H, Wang T, et al. Dysbiosis of Gut Microbiota in Patients With Esophageal Cancer. Microbial Pathogen (2021) 150:104709. doi: 10.1016/j.micpath.2020.104709

84. Liu Y, Lin Z, Lin Y, Chen Y, Peng XE, He F, et al. Streptococcus and Prevotella Are Associated With the Prognosis of Oesophageal Squamous Cell Carcinoma. J Med Microbiol (2018) 67(8):1058-68. doi: 10.1099/ jmm.0.000754

85. Yamamura K, Izumi D, Kandimalla R, Sonohara F, Baba Y, Yoshida N, et al. Intratumoral Fusobacterium Nucleatum Levels Predict Therapeutic Response to Neoadjuvant Chemotherapy in Esophageal Squamous Cell Carcinoma. Clin Cancer Res (2019) 25(20):6170-9. doi: 10.1158/10780432.CCR-19-0318

86. Okereke I, Hamilton C, Reep G, Krill T, Booth A, Ghouri Y, et al. Microflora Composition in the Gastrointestinal Tract in Patients With Barrett's Esophagus. J Thorac Dis (2019) 11(Suppl 12):S1581-7. doi: 10.21037/ jtd.2019.06.15

87. Zhou J, Shrestha P, Qiu Z, Harman DG, Teoh WC, Al-Sohaily S, et al. Distinct Microbiota Dysbiosis in Patients With Non-Erosive Reflux Disease and Esophageal Adenocarcinoma. J Clin Med (2020) 9(7):2162. doi: 10.3390/ jcm9072162

88. Kerr J, Anderson C, Lippman SM. Physical Activity, Sedentary Behaviour, Diet, and Cancer: An Update and Emerging New Evidence. Lancet Oncol (2017) 18(8):e457-71. doi: 10.1016/S1470-2045(17)30411-4

89. Sawada A, Fujiwara Y, Nagami Y, Tanaka F, Yamagami H, Tanigawa T, et al. Alteration of Esophageal Microbiome by Antibiotic Treatment Does Not Affect Incidence of Rat Esophageal Adenocarcinoma. Digestive Dis Sci (2016) 61(11):3161-8. doi: 10.1007/s10620-016-4263-6

90. Zhang K, Dai H, Liang W, Zhang L, Deng Z. Fermented Dairy Foods Intake and Risk of Cancer. Int J Cancer (2019) 144(9):2099-108. doi: 10.1002/ ijc.31959

91. Doron S, Snydman DR. Risk and Safety of Probiotics. Clin Infect Dis (2015) 60 Suppl 2:S129-34. doi: 10.1093/cid/civ085

92. Brown AC, Valiere A. Probiotics and Medical Nutrition Therapy. Nutr Clin Care (2004) 7(2):56-68.

93. Zitvogel L, Daillère R, Roberti MP, Routy B, Kroemer G. Anticancer Effects of the Microbiome and its Products. Nat Rev Microbiol (2017) 15(8):465-78. doi: $10.1038 /$ nrmicro.2017.44

94. Gibson MK, Crofts TS, Dantas G. Antibiotics and the Developing Infant Gut Microbiota and Resistome. Curr Opin Microbiol (2015) 27:51-6. doi: 10.1016/j.mib.2015.07.007

95. Ianiro G, Tilg H, Gasbarrini A. Antibiotics as Deep Modulators of Gut Microbiota: Between Good and Evil. Gut (2016) 65(11):1906-15. doi: 10.1136/gutjnl-2016-312297

96. Rothschild D, Weissbrod O, Barkan E, Kurilshikov A, Korem T, Zeevi D, et al. Environment Dominates Over Host Genetics in Shaping Human Gut Microbiota. Nature (2018) 555(7695):210-5. doi: 10.1038/nature25973

97. David LA, Maurice CF, Carmody RN, Gootenberg DB, Button JE, Wolfe BE, et al. Diet Rapidly and Reproducibly Alters the Human Gut Microbiome. Nature (2014) 505(7484):559-63. doi: 10.1038/nature12820

98. Hildebrandt MA, Hoffmann C, Sherrill-Mix SA, Keilbaugh SA, Hamady M, Chen Y-Y, et al. High-Fat Diet Determines the Composition of the Murine
Gut Microbiome Independently of Obesity. Gastroenterology (2009) 137 (5):1716-24.e1-2. doi: 10.1053/j.gastro.2009.08.042

99. Münch NS, Fang H-Y, Ingermann J, Maurer HC, Anand A, Kellner V, et al. High-Fat Diet Accelerates Carcinogenesis in a Mouse Model of Barrett's Esophagus via Interleukin 8 and Alterations to the Gut Microbiome. Gastroenterology (2019) 157(2):492-506.e2. doi: 10.1053/j.gastro.2019. 04.013

100. Molendijk J, Nguyen T-M-T, Brown I, Mohamed A, Lim Y, Barclay J, et al. Chronic High-Fat Diet Induces Early Barrett's Esophagus in Mice Through Lipidome Remodeling. Biomolecules (2020) 10(5):776. doi: 10.3390/ biom 10050776

101. Hutkins RW, Krumbeck JA, Bindels LB, Cani PD, Fahey G, Goh YJ, et al. Prebiotics: Why Definitions Matter. Curr Opin Biotechnol (2016) 37:1-7. doi: 10.1016/j.copbio.2015.09.001

102. So D, Whelan K, Rossi M, Morrison M, Holtmann G, Kelly JT, et al. Dietary Fiber Intervention on Gut Microbiota Composition in Healthy Adults: A Systematic Review and Meta-Analysis. Am J Clin Nutr (2018) 107(6):965-83. doi: 10.1093/ajcn/nqy041

103. Nobel YR, Snider EJ, Compres G, Freedberg DE, Khiabanian H, Lightdale CJ, et al. Increasing Dietary Fiber Intake Is Associated With a Distinct Esophageal Microbiome. Clin Trans Gastroenterol (2018) 9(10):199. doi: 10.1038/s41424-018-0067-7

104. Lagergren J, Lagergren P. Recent Developments in Esophageal Adenocarcinoma. CA: Cancer J Clin (2013) 63(4):232-48. doi: 10.3322/ caac. 21185

105. Hardikar S, Onstad L, Blount PL, Odze RD, Reid BJ, Vaughan TL. The Role of Tobacco, Alcohol, and Obesity in Neoplastic Progression to Esophageal Adenocarcinoma: A Prospective Study of Barrett's Esophagus. PLoS One (2013) 8(1):e52192. doi: 10.1371/journal.pone.0052192

106. Kant P, Hull MA. Excess Body Weight and Obesity-the Link With Gastrointestinal and Hepatobiliary Cancer. Nat Rev Gastroenterol Hepatol (2011) 8(4):224-38. doi: 10.1038/nrgastro.2011.23

107. Cani PD, Amar J, Iglesias MA, Poggi M, Knauf C, Bastelica D, et al. Metabolic Endotoxemia Initiates Obesity and Insulin Resistance. Diabetes (2007) 56(7):1761-72. doi: 10.2337/db06-1491

108. Le Chatelier E, Nielsen T, Qin J, Prifti E, Hildebrand F, Falony G, et al. Richness of Human Gut Microbiome Correlates With Metabolic Markers. Nature (2013) 500(7464):541-6. doi: 10.1038/nature12506

109. Ley RE, Turnbaugh PJ, Klein S, Gordon JI. Microbial Ecology: Human Gut Microbes Associated With Obesity. Nature (2006) 444(7122):1022-3. doi: $10.1038 / 4441022 a$

110. Liu R, Hong J, Xu X, Feng Q, Zhang D, Gu Y, et al. Gut Microbiome and Serum Metabolome Alterations in Obesity and After Weight-Loss Intervention. Nat Med (2017) 23(7):859-68. doi: 10.1038/nm.4358

111. Sjöström L, Gummesson A, Sjöström CD, Narbro K, Peltonen M, Wedel H, et al. Effects of Bariatric Surgery on Cancer Incidence in Obese Patients in Sweden (Swedish Obese Subjects Study): A Prospective, Controlled Intervention Trial. Lancet Oncol (2009) 10(7):653-62. doi: 10.1016/S14702045(09)70159-7

112. Palombo G, Merone M, Altomare A, Gori M, Terradura C, Bacco L, et al. The Impact of the Intestinal Microbiota and the Mucosal Permeability on Three Different Antibiotic Drugs. Eur J Pharm Sci (2021) 164. doi: 10.1016/ j.ejps.2021.105869

113. Yang ZD, Guo YS, Huang JS, Gao YF, Peng F, Xu RY, et al. Isomaltulose Exhibits Prebiotic Activity, and Modulates Gut Microbiota, the Production of Short Chain Fatty Acids, and Secondary Bile Acids in Rats. Molecules (2021) 26(9):2464. doi: 10.3390/molecules26092464

114. Salgaco MK, Perina NP, Tome TM, Mosquera EMB, Lazarini T, Sartoratto A, et al. Probiotic Infant Cereal Improves Children's Gut Microbiota: Insights Using the Simulator of Human Intestinal Microbial Ecosystem (SHIME(R)). Food Res Int (2021) 143:110292. doi: 10.1016/j.foodres.2021.110292

115. Jing Y, Yu Y, Bai F, Wang L, Yang D, Zhang C, et al. Effect of Fecal Microbiota Transplantation on Neurological Restoration in a Spinal Cord Injury Mouse Model: Involvement of Brain-Gut Axis. Microbiome (2021) 9 (1):59. doi: 10.1186/s40168-021-01007-y

116. Alexander JL, Wilson ID, Teare J, Marchesi JR, Nicholson JK, Kinross JM. Gut Microbiota Modulation of Chemotherapy Efficacy and Toxicity. Nat Rev Gastroenterol Hepatol (2017) 14(6):356-65. doi: 10.1038/nrgastro.2017.20 
117. Wei SC, Duffy CR, Allison JP. Fundamental Mechanisms of Immune Checkpoint Blockade Therapy. Cancer Discov (2018) 8(9):1069-86. doi: 10.1158/2159-8290.CD-18-0367

118. Gopalakrishnan V, Spencer CN, Nezi L, Reuben A, Andrews MC, Karpinets TV, et al. Gut Microbiome Modulates Response to Anti-PD-1 Immunotherapy in Melanoma Patients. Science (2018) 359(6371):97-103. doi: 10.1126/science.aan4236

119. Matson V, Fessler J, Bao R, Chongsuwat T, Zha Y, Alegre ML, et al. The Commensal Microbiome is Associated With Anti-PD-1 Efficacy in Metastatic Melanoma Patients. Science (2018) 359(6371):104-8. doi: 10.1126/science.aao3290

120. Routy B, Le Chatelier E, Derosa L, Duong CPM, Alou MT, Daillere R, et al. Gut Microbiome Influences Efficacy of PD-1-Based Immunotherapy Against Epithelial Tumors. Science (2018) 359(6371):91-7. doi: 10.1126/ science.aan 3706

121. Sivan A, Corrales L, Hubert N, Williams JB, Aquino-Michaels K, Earley ZM, et al. Commensal Bifidobacterium Promotes Antitumor Immunity and Facilitates Anti-PD-L1 Efficacy. Science (2015) 350(6264):1084-9. doi: $10.1126 /$ science.aac4255

122. Vetizou M, Pitt JM, Daillere R, Lepage P, Waldschmitt N, Flament C, et al. Anticancer Immunotherapy by CTLA-4 Blockade Relies on the Gut Microbiota. Science (2015) 350(6264):1079-84. doi: 10.1126/science.aad1329

123. Peled JU, Devlin SM, Staffas A, Lumish M, Khanin R, Littmann ER, et al. Intestinal Microbiota and Relapse After Hematopoietic-Cell Transplantation. J Clin Oncol (2017) 35(15):1650-9. doi: 10.1200/JCO. 2016.70.3348

124. Giamarellos-Bourboulis EJ, Bengmark S, Kanellakopoulou K, Kotzampassi K. Pro- and Synbiotics to Control Inflammation and Infection in Patients With Multiple Injuries. J Trauma (2009) 67(4):815-21. doi: 10.1097/ TA.0b013e31819d979e

125. Shimizu K, Ogura H, Goto M, Asahara T, Nomoto K, Morotomi M, et al. Synbiotics Decrease the Incidence of Septic Complications in Patients With Severe SIRS: A Preliminary Report. Digestive Dis Sci (2009) 54(5):1071-8. doi: $10.1007 /$ s10620-008-0460-2
126. van Minnen LP, Timmerman HM, Lutgendorff F, Verheem A, Harmsen W, Konstantinov SR, et al. Modification of Intestinal Flora With Multispecies Probiotics Reduces Bacterial Translocation and Improves Clinical Course in a Rat Model of Acute Pancreatitis. Surgery (2007) 141(4):470-80. doi: 10.1016/j.surg.2006.10.007

127. Reddy BS, Macfie J, Gatt M, Larsen CN, Jensen SS, Leser TD. Randomized Clinical Trial of Effect of Synbiotics, Neomycin and Mechanical Bowel Preparation on Intestinal Barrier Function in Patients Undergoing Colectomy. Br J Surg (2007) 94(5):546-54. doi: 10.1002/bjs.5705

128. Okada Y, Tsuzuki Y, Hokari R, Komoto S, Kurihara C, Kawaguchi A, et al. Anti-Inflammatory Effects of the Genus Bifidobacterium on Macrophages by Modification of Phospho-I kappaB and SOCS Gene Expression. Int J Exp Pathol (2009) 90(2):131-40. doi: 10.1111/j.1365-2613.2008.00632.x

129. van Nood E, Vrieze A, Nieuwdorp M, Fuentes S, Zoetendal EG, de Vos WM, et al. Duodenal Infusion of Donor Feces for Recurrent Clostridium Difficile. N Engl J Med (2013) 368(5):407-15. doi: 10.1056/NEJMoa1205037

Conflict of Interest: The authors declare that the research was conducted in the absence of any commercial or financial relationships that could be construed as a potential conflict of interest.

Publisher's Note: All claims expressed in this article are solely those of the authors and do not necessarily represent those of their affiliated organizations, or those of the publisher, the editors and the reviewers. Any product that may be evaluated in this article, or claim that may be made by its manufacturer, is not guaranteed or endorsed by the publisher.

Copyright (c) 2021 Zhou, Sun, Luan, Xiao, Yang, Mao, Chen, Zeng, Zhang and Yuan. This is an open-access article distributed under the terms of the Creative Commons Attribution License (CC BY). The use, distribution or reproduction in other forums is permitted, provided the original author(s) and the copyright owner(s) are credited and that the original publication in this journal is cited, in accordance with accepted academic practice. No use, distribution or reproduction is permitted which does not comply with these terms. 